\title{
Identification and Comparative Molecular Docking Analysis of 6, 7, 8, 9-Tetrahydro-2H-11-oxa-2, 4, 10-triaza-benzo[B] fluoren-1-one (RBMS-01) Bounds with DPP4 for Anti-Hyperglycemic Activities
}

\author{
R. BALAJEE* and M. S. DHANARAJAN \\ *Sathyabama University, Chennai 600 119, India \\ Jaya College of Arts and Science, Thiruninravur- 602024, India \\ rbalajeebio@gmail.com
}

Received 12 May 2012 / Accepted 30 May 2012

\begin{abstract}
RBMS-01, a quinoline derivative is a novel dipeptidyl peptidase (DPP4) inhibitor was reported. We have identified this DPP-IV inhibitor in silico as an anti-diabetic agent, the mechanism reveals that the quinoline derivative increases the DPP4 function by degrading GLP-1 activity. Further, the study revealed by replacing the quinoline substitution on the various compounds in a more polar group, these compound shows not only enhancing the binding and functional activity of DPP4, also significantly shows the hERG inhibitory activities. Here, it reveals that the library of compounds constructed based on heterocyclic ring and performed docking using GLIDE, in an order with inhibition scores and compared with already existing drugs. An active site of these compounds was determined termed as the lead molecule, the molecule formula is, 6,7,8,9-Tetrahydro- $2 \mathrm{H}$-11-oxa2,4,10-triaza-benzo[b]fluoren-1-one, (RBMS-01) emerged as a potent, DPP4 inhibitor that displayed. The pharmacophoric analysis also shows that the novel inhibitor have better activity than the other inhibitors.
\end{abstract}

Keywords: Docking, Synthetic molecule, DPP4, Quinoline, Pharmacophore analysis, GLP1, GIP

\section{Introduction}

Progressive in nature and characterized by high circulating levels of glucose resulting from insulin resistance and impaired insulin secretion, type 2 diabetes mellitus causes significant morbidity and mortality and results in considerable burden on healthcare resources (http://www.who.int/mediacentre/factsheets/fs312/en/index.html). Current treatment strategies for type 2 diabetes mellitus have only limited long-term efficacy and tolerability given the progressive nature of the disease ${ }^{1}$. A breakthrough was the discovery that postprandial plasma glucose concentrations are regulated by the incretin hormones glucagon-like peptide (GLP) 1 and glucose-dependent insulinotropic peptide (GIP), which sensitize $\beta$-cells to glucose stimulation, leading to increased intracellular cAMP concentrations 
in $\beta$-cells and an accelerated and augmented insulin response to absorbed glucose ${ }^{2,3}$. The insulinotropic actions of GLP-1 and GIP are glucose-dependent; therefore, the risk of hypoglycemia is minimized. In healthy subjects, the insulinotropic effect of GLP-1 accounts for 50 to $70 \%$ of the insulin response to an oral glucose load, but the associated "incretin effect" is often markedly reduced in patients with type 2 diabetes ${ }^{2}$. This quick inactivation process leads to an apparent half-life of 60-90s for GLP-1 (7-36) and there is evidence that less than $50 \%$ of released active GLP-1 (7-36) can reach circulation because of this natural degradation mechanism ${ }^{4}$. It is therefore apparent that a DPP4 inhibitor can prevent degradation of and lead to potentiation of GLP-1, which will further improve glucose and insulin homeostasis ${ }^{5}$.

\section{Experimental}

The protein data bank (PDB) is a repository for the 3-D structural data of large biological molecules, such as proteins and nucleic acids. The data, typically obtained by $\mathrm{x}$-ray crystallography or NMR spectroscopy and submitted by biologists and biochemists from around the world, are freely accessible on the internet via the websites of its member organisations (PDBe, PDBj and RCSB). The PDB is overseen by an organization called the worldwide protein data bank, wwPDB.

\section{Dipeptidyl peptidase}

DPP4 is a non-classical, sequence-specific serine protease that is ubiquitously expressed throughout the body (Figure 1). Membrane bound DPP4 is especially highly expressed in the endothelium of the capillary bed in close proximity to intestinal $L$-cells where GLP- 1 is secreted. There is also a soluble form of DPP4 circulating in plasma, although this form plays little role in the cleavage of GLP-1 $1^{6,7}$.

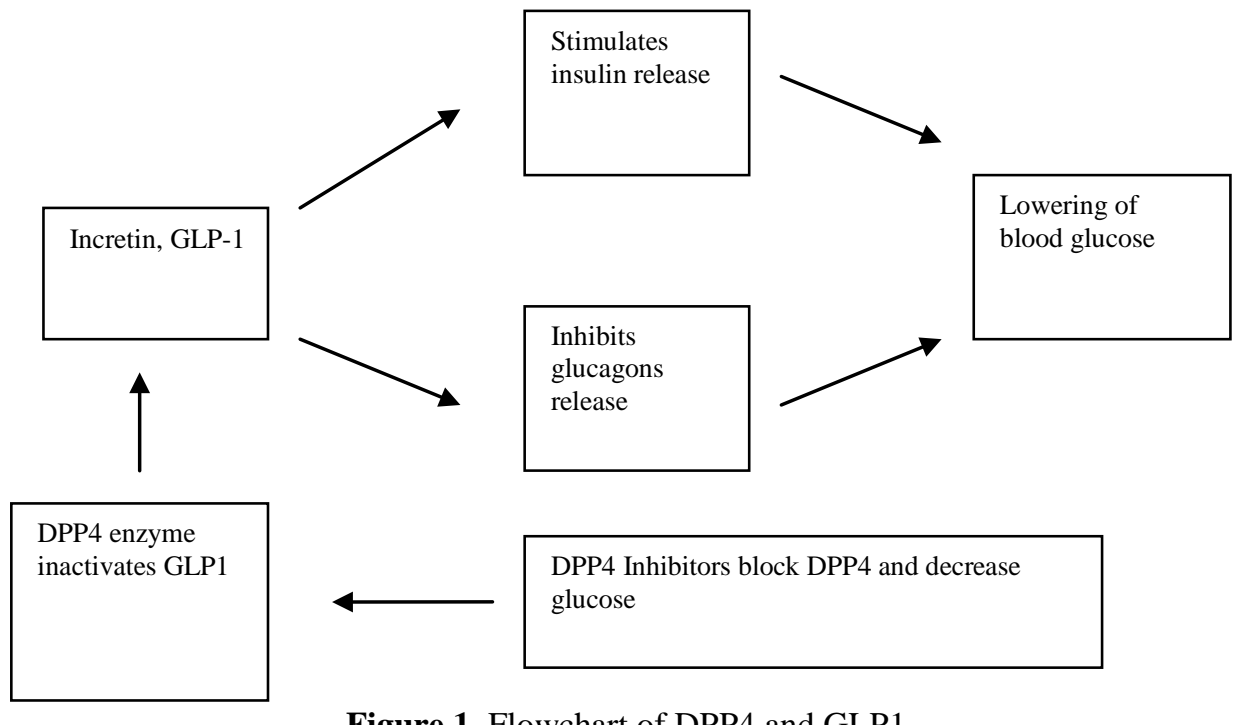

Figure 1. Flowchart of DPP4 and GLP1

Clinical studies shows that insulin resistance states and levels of DPP4 expressions in pancreatic alpha and beta cell, suggesting that DPP4 has a major role in anti-hyperglycemic effect. Blocking the GLP-1 could enhance the rate of inhibitors, which is a characteristic of type II diabetes ${ }^{4}$. 
Since then, many drugs have been synthesized for targeting DPP4, which is very challenging due to form of the binding site containing a highly serine residues. Earlier studies reports here, that the heteroaromatic group plays a very major role towards the binding affinity. However, all of these compounds also displayed high levels of the human ether-a-go-go related gene (hERG) and sodium channel inhibition. To minimize this activity, the novel compound is derivative of quinoline group is discovered. This compound represents an advanced lead with drug-like properties as a potential treatment for type II diabetes.

\section{PyMol (http://www.pymol.org)}

PyMOL is an open-source, user-sponsored, molecular visualization system created by Warren Lyford DeLano and commercialized by DeLano Scientific LLC, which is a private software company dedicated to creating useful tools that become universally accessible to scientific and educational communities. It can produce high quality 3D images of small molecules and biological macromolecules, such as proteins. According to the author, almost a quarter of all published images of 3D protein structures in the scientific literature were made using PyMOL.

\section{Ligand preparation}

The compounds were obtained from Interbioscreen, Russia (IBS) and natural compounds from various databases. The inhibitory molecules were taken and prepared and modified for assigning the appropriate bond orders by using Ligprep module and also study was enhanced by using Qikprop to predict biological and physiological properties. These modules were installed in Maestro, Schrodinger Software. Out of these, the better compounds are filtered by using GLIDE score and Energy values. It is modified using ligprep module of the Maestro application, Schrodinger Inc, by assigning the appropriate bond orders manually. Each ligand was prepared manually with a full minimization with force field of the parameters using OPLS (Optimized potential for liquid simulations) ${ }^{8}$ to eliminate bond length and angles biased from the crystal structure. The multiple structure for each ligand (Figure 2) were produced with different combinations, so the protonation states i.e., ionization states were exist to involve in any physiological condition. This operation can be performed using ionizer in Ligprep. Ligand, functions optimally in the $\mathrm{pH}$ range of 7. One or more forms of different conformations were produced to interact more strongly with the respective binding site. Tautomeric activity was also identified for the particular inhibitor.<smiles>[Y9]n1cnc2c(oc3nc4c(cc32)CCCC4)c1=O</smiles><smiles>CCn1ccc2[nH]c3c(c2c1=N)CCC(P)C3</smiles><smiles>[Y9]n1c(CCN2C(=O)c3ccncc3C2=O)nc2cc([Hg])ccc21</smiles>

RBMS-03<smiles>[Y3]c1cc2nc3n(c2cc1Br)C([R3])NCC3</smiles>

RBMS-04 
<smiles>Cc1nc(Nc2nc3cnccc3o2)ncc1C(=O)O</smiles><smiles>[R]C1C(C#N)=C(N)Oc2cc3c(cc21)OCO3</smiles>

RBMS-07<smiles>[R3]c1ccc2c(c1)nc(COCc1ccc(Cl)nc1)n2[Y]</smiles>

RBMS-09<smiles>Cc1nc(Nc2cc3ncccc3o2)ncc1C(=O)O</smiles><smiles>[R]S(=O)(=O)N1CN2CCN(CC2)C1</smiles>

RBMS-08<smiles>C=C(NC)N1CCc2nc[nH]c2C1[10SH]</smiles>

RBMS-10

Figure 2. Structure of Synthesized Ligand Molecules

\section{Macromolecular preparation}

The coordinates for all the proteins obtained from Research Colloboration for Structural Bioinformatics (RCSB, www.rcsb.org) (PDB ID 3NOX). The structure was derived from RCSB in which the A Chain shows the binding site for the ligand. In the present study has considered only one ligand. The complex was prepared by the module protein preparation wizard, where hydrogens were added automatically and refinement of the structure was also done. Water molecules were removed for the protein-ligand interactions and bond orders were re-assigned. The structure was minimized to a Root mean Square deviation of $0.30 \AA$. The atomic coordinates were processed using Swiss PDB viewer to analyze protein structures (http://spdbv.vital-it.ch/).

\section{Macromolecular refinement using Ramachandran plot analysis}

The picture (Figure 3) shows the result as

No. of residues in favoured region : $676(93.6 \%)$

No. of residues in allowed region : $45(6.2 \%)$

No. of residues in outlier region : $\quad 1(0.1 \%)$

\section{Ligand docking parameters}

The ligand was geometrically optimized and prepared the initial coordinates for docking process. The docking can be used in two approaches i.e., by having ligand as flexible and receptor is rigid or ligand is rigid and receptor is flexible. The later approach i.e., receptor rigid option is used for the present study, with the help of GLIDE docking approach.

The ligand docking was performed by the receptor grid generation; for that purpose we have used the next generation target of dipeptidyl peptidase 4 protein. During the grid 
generation, no Vander Waals radius sampling was done and the partial charge cut-off has been taken as 0.25 and no constraints were applied. The location of peptide substrate has taken as binding site for all the other ligands for docking.
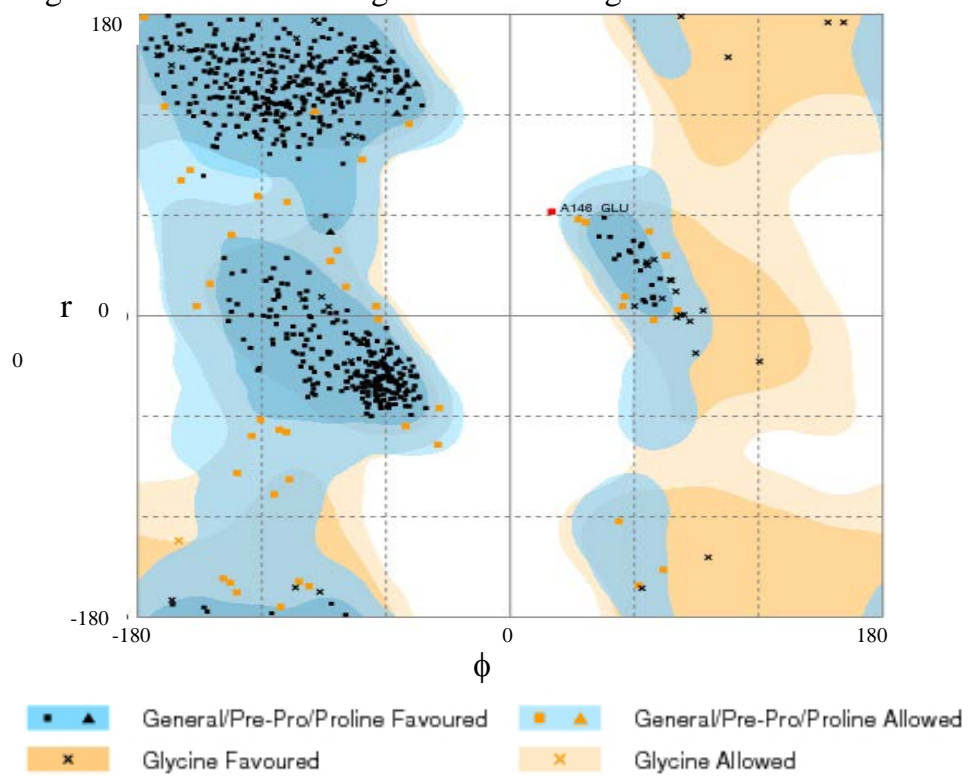

Figure 3. Ramachandran Plot analysis using RAMPAGE (source:http://mordred.bioc.cam.ac.uk)

Results and Discussion

Virtual screening

After screening the library of taken natural and synthetic compounds, the score is sorted according to the High Throughput Virtual Screening (HTVS). The study details obtaining the potential inhibitors were selected according to the Glide Energy from the library of compounds also reflects that synthetic compounds had better activity (Table 1 and Figure 4).

The GLIDE docking method is applied to various inhibitors to build an affinity model to the dipeptidyl peptidase. The training sets of different inhibitors are generated by scoring functions. It was characterized by orientations and Hydrogen bond positions. According to the energy values, the synthetic molecule RBMS-01 shown better activity with low root mean square (RMS) deviations of $0.30 \AA$. Further, it extended by using induced fit approach which described below.

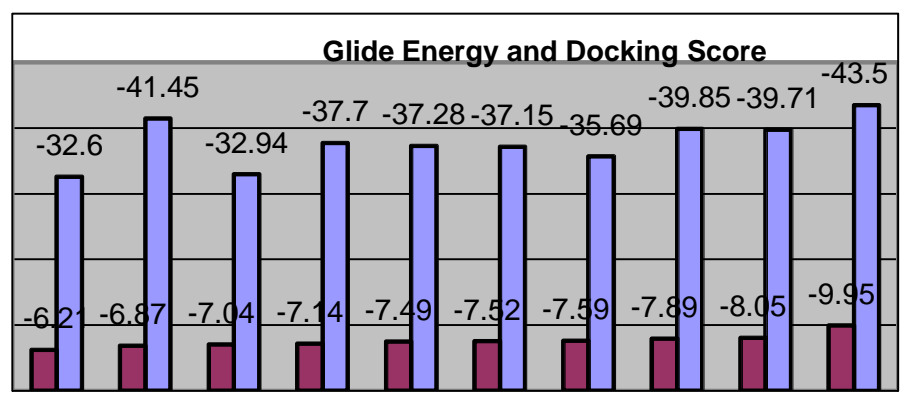

Figure 4. Glide energy and docking score 
To make a better understanding of the binding mode, we were able to obtain x-ray crystal structures of both the DPP4 apo enzyme and DPP4 bound as a co-crystal with an inhibitor in the active site ${ }^{9}$. Consistent with earlier reports, the DPP4 binding pocket is located at the interface of the $\alpha / \beta$-hydrolase and $\beta$-propeller domains ${ }^{10}$. The catalytic triad (S630, H740 and D708), which is highly conserved in the DPP4 gene family (including DPP8 and DPP9), resides in the $\alpha / \beta$-hydrolase domain. On the other hand, E205 and E206, which typically interact with the $N$-terminus of DPP substrates, are located in the $\beta$-propeller domain, which is less conserved across the DPP4 gene family ${ }^{11}$. Result of Vander Waals (Evdw), Electrostatic (Ecoul), Docking score and their energy values after HTVS, GLIDE docking are shown in Table 1 . Here, RBMS-01 shows better activity and interactions compared with other compounds.

Table 1. High Throughput Screening (HTVS) of synthetic compounds

\begin{tabular}{cccccc}
\hline S. No & Inhibitor & Evdw & Ecoul & Energy & Docking Score \\
\hline 1 & RBMS-01 & -14.93 & -28.20 & -43.50 & -9.95 \\
2 & RBMS-02 & -12.50 & -27.30 & -39.71 & -8.05 \\
3 & RBMS-03 & -22.55 & -17.29 & -39.85 & -7.89 \\
4 & RBMS-04 & -11.66 & -24.03 & -35.69 & -7.59 \\
5 & RBMS-05 & -18.89 & -18.26 & -37.15 & -7.52 \\
6 & RBMS-06 & -21.23 & -16.05 & -37.28 & -7.49 \\
7 & RBMS-07 & -15.11 & -22.59 & -37.70 & -7.14 \\
8 & RBMS-08 & -13.13 & -19.81 & -32.94 & -7.04 \\
9 & RBMS-09 & -17.78 & -23.68 & -41.45 & -6.87 \\
10 & RBMS-10 & -13.04 & -19.56 & -32.60 & -6.21 \\
\hline
\end{tabular}

GLP-1, which is secreted in a nutrient-dependent manner, stimulates glucose-dependent insulin secretion and regulates glycemia. However, half-life of active GLP-1 is very short due to degradation by DPP-IV and the actions of GLP-1 do not last long. Then, development of DPP-IV inhibitors has been active worldwide, which is expected to control blood glucose levels by enhancement of the action of GLP-1 for the treatment of diabetes. The development of new agents to control postprandial glucose excursions is considered to be an essential objective for the management of type 2 diabetes. In a course of research of novel DPP-IV inhibitors were discovered from the series of synthetic compound. A recent focus of research of DPP-IV inhibitors showed evidence through in silico analysis, aims with physicochemical properties. A quinoline derivative improves the glycemic control, thus, RBMS-01 might be a novel potent DPP-IV inhibitor with an anti-hyperglycemic activity.

\section{Induced fit docking}

The aim of the present study indicates that the ligands were docked into binding site of the receptor where the receptor is rigid and the ligand is free to move by using the induced fit approach obtaining the filtered compounds from virtual screening method. The macromolecule crystal structure shows the critical hinging and displays more conformations; it may allow side chain modeling ${ }^{12}$. The conformations show that many alterations in the receptor and it is more closely conforms and fit to the ligands. The purpose of docking is to find the affinity between the macromolecular and ligand complex. Further, it shows the binding between the ligands into a rigid receptor, which assumes the correct one with low energy values. The purpose of this method is to eliminate the steric clashes and then the appropriate interaction will be resulted ${ }^{13}$. The IFD docking was performed from least energy 
after series of filtration from HTVS and standard precision using GLIDE docking. The synthesized compound, RBMS-01 was preceded and it recorded $-45.72 \mathrm{kcal}$ as least energy. This reflects by sampling of the receptor degree of freedom and a minimization of the receptor-inhibitor complex for many different receptor poses and it is attempted to identify low free energy conformation of the each complex. Many researchers demonstrated the importance of ligand binding with protein ${ }^{14}$. This better binding affinity witnessed that this novel synthetic molecule have an interaction with macromolecule. Serine 630 and glutamic acid 205 and 206 appear a potential site for anti-hyperglycemic activity.

\section{Interactional analysis}

The macromolecule dipeptidyl peptidase was docked by using induced fit docking with first 2 novel synthetic molecules and (RBMS-01 and RBMS-02) and natural compounds, it was found that synthetic molecule RBMS-01 have high significant affinity producing an evidence of glide energy value with other molecules (Table 2). The comparative studies also mentioned in Figure 5. Table 2 describes about the 2 synthetic compounds and 2 natural compounds interactional analysis between ligand and macromolecule where serine, glutamate shows the better activity.

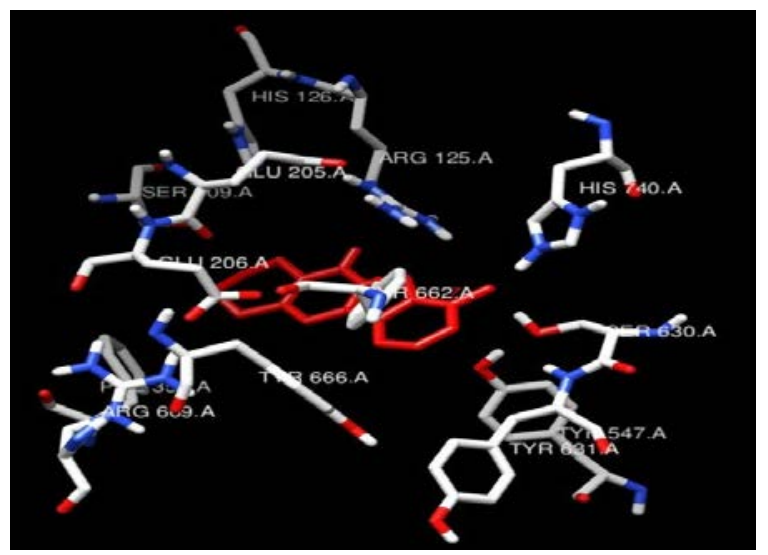

Figure 5.The interaction between macromolecule and ligand molecule displayed, Ser 630 and Glu 205 and 206 positions makes more active

Table 2. Comparison of docking score of synthetic with natural compounds

\begin{tabular}{cccc}
\hline S. No. & Inhibitors & Energy Score & Glide Score \\
\hline 1 & RBMS-01 & -45.72 & -7.49 \\
2 & RBMS-02 & -44.35 & -6.39 \\
3 & Bereberine & -39.24 & -5.00 \\
4 & Metformin & -34.05 & -4.60 \\
\hline
\end{tabular}

From the analysis of the inhibitor bound to DPP4, it is clearly states that the quinoline derivative group fits tightly into the hydrophobic pocket and also the amino group has some interaction with glutamic acid 205 and 206. The amine also exhibited hydrogen interaction with tyrosine 662. These key interactions anchor the molecule into the DPP4 binding site. With the observation of this compounds, this projects to a large solvent-exposed area and can occupy, shows many conformation of different groups including with polar functionality. Unlike the already existing inhibitors, here (Figure 6) the serine 630 from the catalytic triad plays a crucial role in binding and combine with the fact that most of the key contacts 
with subsequent binding energy from this inhibitor within the active site of DPP4 derives from residues in the $\beta$-propeller domain. Result of docking score and their energy values after performing induced fit docking is shown in Table 3.

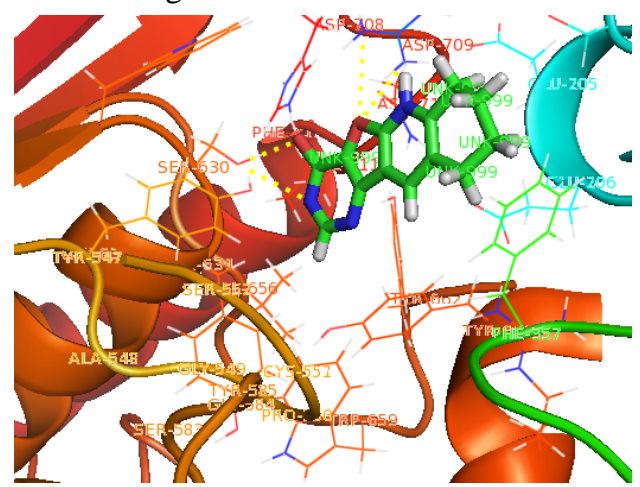

Figure 6. Ligand binding in ribbon model, shows ser 630 plays a important role by interacting directly with nitrogen compound

Table 3. Pharmacophore Analysis

\begin{tabular}{cccc}
\hline Residue & D - H - A & Residue & Distance $\AA$ \\
\hline Unk & O - H - OG & Ser 630 & 2.56 \\
Unk & N2 - H - OG & Ser 630 & 3.03 \\
Arg 125 & NH2 - H - O1 & Unk & 3.34 \\
Arg 125 & NH2 - H - O2 & Unk & 3.09 \\
\hline
\end{tabular}

ADME or pharmacokinetic predictions of the best fit molecules

Various predictive binding models for hERG have been developed and based on in silico modeling predictions for hERG channel blockers, molecular features that may contribute to increased hERG inhibitory activity include hydrophobicity, high molecular weight, the presence of a basic group such as an amine and a V-shape topology arising from ring-linker arrangements $^{15-17}$.

The ligands with the comparable scores with other molecules were subjected to predict pharmacokinetic properties using the QikProp module of the software. QikProp settings determine which molecules are flagged as being dissimilar to other 95\% of the known drugs. Predicted significant ADME properties such as permeability through the predicted log IC50 value for blockage of $\mathrm{K}^{+}$channels (QPlogHERG), QikProp predicted gut-blood barrier and no violations of Lipinski's rule of five are reported here. The predicted property of docked compound RBMS-01 and information about ligand is given in Table 4. The drug molecule which satisfies Lipinski rule of 5, includes molecular weight, hydrogen bond donors and acceptors.

Table 4. Properties of ligand molecule

\begin{tabular}{ll}
\hline Contents & Values \\
\hline Compound Name & RBMS-01 \\
Molecular Weight & 241.25 \\
Octanol/Water & 1.824 \\
Log IC 50 & -3.874 \\
Lipinski’s violations & Nil \\
QlogP MDCK & 332 \\
\hline
\end{tabular}




\section{Derivatives of the parent molecule}

The compound RBMS-01 produces good docking and energy scores when compare to other compounds. The RBMS-01-DER-01 is derived from RBMS-01 by methylation in the $1^{\text {st }}$ position displayed in Figure 9a. The RBMS-01-DER-2 compound also shows the better activity which displayed in Figure 9b. While comparing to RBMS-01-DER-1 and RBMS01-DER-2 the GLIDE score and energy values are scored very least by using high throughput virtual screening (HTVS) RBMS-01-DER-1 stabilizes the energy (Table 5) also it was taken and confirmed using Induced fit model (IFD) showing that RBMS-01-DER1 have the stability and better interaction towards the DPP4. The results were mentioned in Table 6. Then Figure 7 and Figure 8 show the interaction with taken derivative.

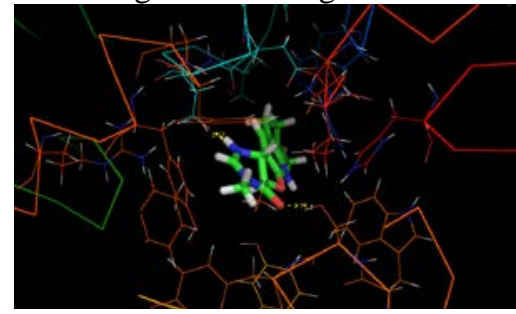

Figure 7. Interaction of RBMS-01DER1 with DPP4<smiles>CC(C)CCn1cnc2c(oc3nc4c(cc32)CCCC4)c1=O</smiles>

RBMS-der-01

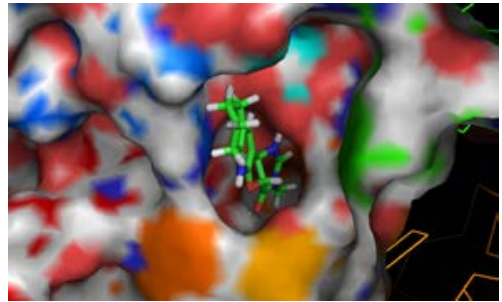

Figure 8. Hydrophobic interaction RBMS-01-DER-1 with DPP4<smiles>COc1cccc(NC(=O)CN2N=Nc3c4nc5c(c3N2O4)CCC5)c1</smiles>

RBMS-der-02

Figure 9. Structure of Derived Compound from RBMS-01

(a) RBMS-01-DER-1; (b) RBMS-01-DER-2.

(The methyl group derived in $1^{\text {st }}$ position of the ring. It shows the better activity comparatively)

Table 5. Docking of derived compounds

\begin{tabular}{ccc}
\hline \multirow{2}{*}{ Compound } & \multicolumn{2}{c}{ HTVS } \\
\cline { 2 - 3 } & Energy & G score \\
\hline RBMS-01-DER-1 & -38.19 & -5.91 \\
RBMS-01-DER-2 & -34.13 & -4.78 \\
\hline
\end{tabular}

Table 6. Induced fit docking of derived compounds

\begin{tabular}{ccc}
\hline \multirow{2}{*}{ Protein } & \multicolumn{2}{c}{ Induced fit docking } \\
\cline { 2 - 3 } & Glide Energy & G Score \\
\hline RBMS-01-DER-1 & -7.14 & -39.87 \\
RBMS-01-DER-2 & -5.32 & -33.14 \\
\hline
\end{tabular}

\section{Conclusion}

Here, we have developed a novel series of inhibitor as potent DPP4. By representing this molecule displayed enhanced DPP4 inhibitory activity, as well as markedly reduced hERG activity. Polarity of the compounds was further modified to improve, the balance of offtarget activity, leading to amide-containing inhibitors possessing drug-like properties. The Macromolecule which observed shows the constant and stable with the series of inhibitors. 
Further, an evaluation of these compounds through this approach shows better drug-like properties and further validated as potential treatments for type II diabetes.

\section{Acknowledgement}

I extend my sincere thanks to Dr. D. Velmurugan, HOD and Co-ordinator, Bioinformatics Infrastructure Facility (BIF), Dept. of Biophysics and crystallography, University of Madras, Guindy Campus, Chennai, encouraged and gave me a continuous support by providing a Schrodinger Version 10 software.

\section{References}

1. Bolen S, Feldman L, Vassy J, Wilson L, Yeh H J, Marinopoulos S, Wiley C, Selvin E, Wilson R, Bass E B and Brancati F L, Ann Intern Med., 2007, 147, 386.

2. Meier J J and Nauck M A, Curr Diab Rep., 2006, 6, 194.

3. $\quad$ Todd J F, Diabet Med., 2007, 24(3), 223-232.

4. Holst J J, Hansen L, Deacon C F and Orskov C, Endocrinology, 1999, 140(11), 5356-5363.

5. Nagakura T, Yasuda N, Yamazaki K, Ikuta H, Yoshikawa S, Asano O and Tanaka I, Biochem Biophys Res Commun., 2001, 284, 501-506.

6. Marguet D, Baggio L, Kobayashi T, Bernard A M, Pierres M, Nielsen P F, Ribel U, Watanabe T, Drucker D J and Wagtmann N, Proc Natl Acad Sci U.S.A, 2000, 97(12), 6874-6879.

7. Durinx C, Lambeir A M, Bosmans E, Falmagne J, Berghmans R, Haemers A, Scharpe S and De Meester I, Eur J Biochem., 2000, 267(17), 5608-5613.

8. Jorgenson W L, Maxwell D S and Tirado-Rives J, J Am Chem Soc., 1996, 118(45), 11225-11236.

9. Iwaki-Egawa S, Watanabe Y, Kikuya Y and Fujimoto Y, J Biochem., 1998, 124(2), 428-433.

10. Metzler W J, Yanchunas J, Weigelt C, Kish K, Klei H E, Xie D, Zhang Y, Corbett M, Tamura J K, He B, Hamann L G, Kirby M S and Marcinkeviciene J, Protein Sci., 2008, 17(2), 240-250.

11. Thoma R, Loffler B, Stihle M, Huber W, Ruf A and Hennig M, Structure, 2003, 11(8), 947-959.

12. Engel M, Hoffmann T, Wagner T, Wermann M, Heiser U, Kiefersauer R, Huber R, Bode W, Demuth H U and Brandstetter H, Proc Natl Acad Sci U.S.A, 2003, 100, 5063-5068.

13. Taylor R D, Jewsbury P J and Essex J W J, Comp Aided Mol Des., 2002, 16, 151-166.

14. Meher B R, Satish Kumar M V and Bandyopadhyay P, Indian J Phy., 2009, 83, 81.

15. Aronov A M, Drug Discov Today, 2005, 10(2), 149-155.

16. Song $M$ and Clark M, $J$ Chem Inf Model, 2006, 46, 392-400.

17. Vaz R J, Li Y and Rampe D, Prog Med Chem., 2005, 43, 1-18. 